\title{
Modulation of Visually Evoked Cortical fMRI Responses by Phase of Ongoing Occipital Alpha Oscillations
}

\author{
René Scheeringa, ${ }^{1,2 \star}$ Ali Mazaheri, ${ }^{3,4 *}$ Ingo Bojak, ${ }^{3}$ David G. Norris, ${ }^{3}$ and Andreas Kleinschmidt ${ }^{1,2}$ \\ ${ }^{1}$ INSERM Unité 992 Cognitive Neuroimaging, and ${ }^{2}$ Commissariat à l'Energie Atomique, Direction des Sciences du Vivant, Institut d'Imagerie Biomédicale, \\ NeuroSpin, F-91191 Gif-sur-Yvette, France, ${ }^{3}$ Donders Institute for Brain, Cognition and Behaviour, Radboud University of Nijmegen, NL-6500 HB \\ Nijmegen, The Netherlands, and ${ }^{4}$ Center for Mind and Brain, University of California, Davis, Davis, California 95618
}

Using simultaneous electroencephalography as a measure of ongoing activity and functional magnetic resonance imaging (fMRI) as a measure of the stimulus-driven neural response, we examined whether the amplitude and phase of occipital alpha oscillations at the onset of a brief visual stimulus affects the amplitude of the visually evoked fMRI response. When accounting for intrinsic coupling of alpha amplitude and occipital fMRI signal by modeling and subtracting pseudo-trials, no significant effect of prestimulus alpha amplitude on the evoked fMRI response could be demonstrated. Regarding the effect of alpha phase, we found that stimuli arriving at the peak of the alpha cycle yielded a lower blood oxygenation level-dependent (BOLD) fMRI response in early visual cortex (V1/V2) than stimuli presented at the trough of the cycle. Our results therefore show that phase of occipital alpha oscillations impacts the overall strength of a visually evoked response, as indexed by the BOLD signal. This observation complements existing evidence that alpha oscillations reflect periodic variations in cortical excitability and suggests that the phase of oscillations in postsynaptic potentials can serve as a mechanism of gain control for incoming neural activity. Finally, our findings provide a putative neural basis for observations of alpha phase dependence of visual perceptual performance.

\section{Introduction}

The dominant oscillatory electroencephalography (EEG) activity during resting wakefulness is observed to be $\sim 10 \mathrm{~Hz}$ and is commonly referred to as the alpha rhythm (Berger, 1929). The amplitude of alpha activity has been suggested to play an important role in gauging the brain's capacity to process information (Klimesch et al., 2007; Mazaheri and Jensen, 2010). Evidence for this has been found in a number of studies that have shown a reduction in the amplitude of alpha activity in regions processing taskrelevant stimulus features, accompanied by an increase in alpha amplitude in regions not required for the task (Thut et al., 2003, 2006; Jokisch and Jensen, 2007; Medendorp et al., 2007; Rihs et al., 2007; Romei et al., 2008a,b). Further studies have shown that this latter increase in posterior alpha activity reduces visual discrimination abilities (van Dijk et al., 2008; Zhang et al., 2008).

Compared with the effect of the amplitude (or power) of alpha activity, the evidence regarding an impact of oscillation phase on neural processing and perceptual performance has remained rather sparse. Several studies have successfully linked differences in the shape or power of poststimulus activity to the prestimulus

\footnotetext{
Received Sept. 7, 2010; revised Dec. 5, 2010; accepted Dec. 10, 2010.

This work was supported by the Agence Nationale de la Recherche. A.M. was supported by a Rubicon grant from The Netherlands Organization for Scientific Research.

We dedicate this paper to Rolf Kötter, who passed away during the writing of this manuscript.

*R.S. and A.M. contributed equally to this work.

Correspondence should be addressed to Ali Mazaheri or René Scheeringa, Donders Institute for Brain, Cognition and Behaviour, Radboud University of Nijmegen, P.0. Box 9101, NL-6500 HB, Nijmegen, The Netherlands. E-mail: Ali.Mazah@gmail.com or Rene.Scheeringa@gmail.com.

DOI:10.1523/JNEUROSCI.4697-10.2011

Copyright $\odot 2011$ the authors $\quad 0270-6474 / 11 / 313813-08 \$ 15.00 / 0$
}

phase of ongoing alpha activity (Bechtereva and Zontov, 1962; Dustman and Beck, 1965; Makeig et al., 2002; Barry et al., 2003). However, this relationship has remained controversial because of the difficulties in unambiguously separating evoked responses from the ongoing activity (Kruglikov and Schiff, 2003; Shah et al., 2004; Yeung et al., 2004; Mazaheri and Jensen, 2006; Klimesch et al., 2009; Risner et al., 2009; Ritter and Becker, 2009).

Even though it was suggested nearly 80 years ago that cortical responsiveness undergoes cyclic changes (Bishop, 1932), only a few studies since then have shown an influence of the phase of the ongoing activity on the visual processing of stimuli (Callaway and Yeager, 1960; Dustman and Beck, 1965; Nunn and Osselton, 1974; Varela et al., 1981). Two recent EEG studies using periliminal stimuli (Busch et al., 2009; Mathewson et al., 2009) found a link between the phase of the prestimulus alpha activity and conscious perception (for review, see Wyart and Sergent, 2009). However, a direct demonstration of phase-dependent local cortical response properties has not yet been sufficiently well achieved. In the present study, we used simultaneous EEG (as a time-resolved measure of ongoing oscillatory activity phase) and functional magnetic resonance imaging (fMRI) (as a spatially resolved bulk measure of the local stimulus-driven response) to examine how the evoked cortical blood oxygenation leveldependent (BOLD) response is affected by the amplitude and phase of ongoing alpha activity at the onset of a brief visual stimulus. For amplitude, but not for phase, it was important to model pseudo-trials without visual stimulation to account for the intrinsic, nonstimulus-driven relationship between occipital alpha activity and the BOLD signal that has been demonstrated in a number of studies (Goldman et al., 2002; Gonçalves et al., 2006; 
A

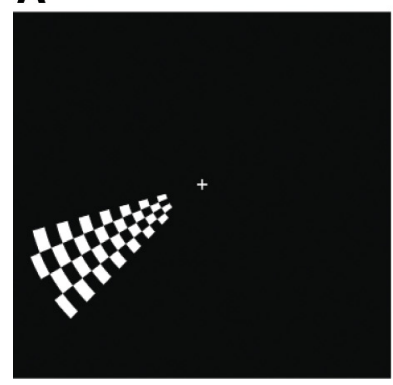

B

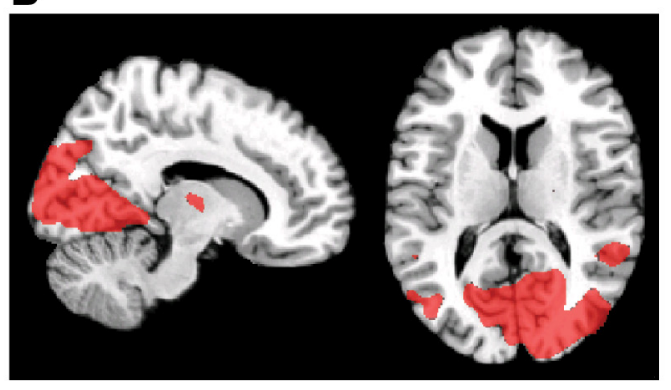

C

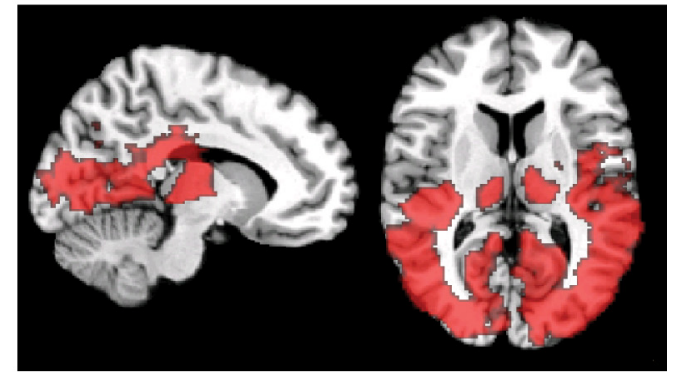

Figure 1. Experimental paradigm and the associated BOLD responses. $\boldsymbol{A}, \mathrm{A}$ wedge-shaped stimulus was presented at random intervals for $17 \mathrm{~ms}$ in the lower-left visual field quadrant. Subjects were asked to press a button whenever the continuously present central fixation cross turned green, which occurred at unpredictable intervals and was uncorrelated with peripheral stimulation. $\boldsymbol{B}$, Regions activated in response to the wedge-shaped stimulus. $\boldsymbol{C}$, Regions activated during the reported color change of the fixation cross. Activations in $\boldsymbol{B}$ and $\boldsymbol{C}$ are displayed at $p<0.001$, uncorrected (second-level analysis, general linear model).

Laufs et al., 2006; Ritter et al., 2009; Scheeringa et al., 2009; Yuan et al., 2010).

\section{Materials and Methods \\ Subjects}

Eighteen subjects ( 14 female; 4 male; mean age, 25 years; range, 18-65), with no history of psychiatric or neurological disorders, participated in the simultaneous EEG/fMRI sessions. All had normal or corrected-tonormal vision. Before the start of the experiment, written informed consent was obtained from each subject. The experiment was approved by a local ethical committee (CMO region Arnhem-Nijmegen, The Netherlands). One female subject was excluded from further analyses because of anxiety and consecutive excessive movement during scanning.

\section{Experimental paradigm}

To ensure subjects' cooperation and alertness, they were asked to fixate a central fixation cross (width, $0.75^{\circ}$ ) and report by a button press whenever it turned green. During each session, the fixation cross changed to green for $300 \mathrm{~ms}$ eight times at pseudorandom moments. Not related to this central incidental task, short (17 ms), wedge-shaped, black-andwhite checkerboard visual stimuli were presented in the lower left corner of the visual field (angle of wedge, $32.7^{\circ}$; visual angle width, $10.4^{\circ}$; starting $3.8^{\circ}$ outside the center of fixation). These peripheral stimuli required no subject response. The stimulus and fixation mark are depicted in Figure $1 \mathrm{~A}$. In addition to the short but strong peripheral visual stimuli as experimental trials of interest, we designed pseudo-trials without such stimulation to estimate the intrinsic correlation of alpha amplitude with the occipital BOLD signal. For these trials, no visual stimulus was presented apart from the central fixation cross that was always present. Forty trials of each condition occurred at random intervals ranging from 3 to $7 \mathrm{~s}$ according to a uniform chance distribution. Six sessions of $\sim 6.5 \mathrm{~min}$ were recorded with this paradigm.

\section{EEG data acquisition}

EEG data were recorded with an MRI-compatible cap equipped with carbon-wired Ag/AgCL electrodes (Easycap) from 61 scalp positions according to the international $10-10$ system. One additional electrode was placed under the right eye to record eye movements. The reference electrode was placed at FCz. Two MRI-compatible EEG amplifiers (BrainAmp MR, Brain Products) with a built-in $250 \mathrm{~Hz}$ low-pass analog hardware filter were used. The EEG was recorded with a $10 \mathrm{~s}$ time constant and continuously sampled at $5 \mathrm{kHz}$. EEG recordings were performed with Brain Vision Recorder software (Brain Products).

\section{EEG preprocessing}

The EEG data were corrected for gradient and pulse artifacts along the lines described by Allen et al. $(1998,2000)$ using Vision Analyzer (Brain Products). To denoise the EEG data further, we applied Infomax (standard, not extended) independent component analysis (ICA) (Bell and Sejnowski, 1995) on all of the 8-12 Hz bandpass-filtered (fourth-order Butterworth filter) concatenated trials (2 s long) using the algorithm implemented in EEGLab 5.03 (Delorme and Makeig, 2004). For each subject, a single alpha component was identified based on rightlateralized topography and the presence of an alpha peak in the power spectrum when the unmixing weights were applied to unfiltered data. To quantify the amount of ICA-related reduction of noise and other neural sources in the mixed sensor signal, we calculated the percentage of variance explained by the selected component in the data (filtered in the alpha band) for the channel selected for calculating the prestimulus phase and amplitude. On average, the selected component explains 14\% of the biologically generated variance, indicating that ICA application results in a greatly enhanced selectivity for the source of interest. Our logic in data analysis closely followed the suggestions outlined for EEG/ fMRI investigations of trial-by-trial variability in Debener et al. (2006). The accordingly identified single components from all subjects were projected back to channel level. To approximately indicate where the source of this alpha component was localized, we conducted a dipole analyses in BESA2000 (v 5.2; BESA) using a four-shell elliptical head model (Berg and Scherg, 1994). For each individual subject, a genetic algorithm (standard in BESA) was used to fit a single dipole to the scalp topography of the subject's alpha component's mixing weights.

\section{EEG analysis}

Instantaneous phase and amplitude estimates. Phase was calculated by discrete Fourier transforms (DFTs) for the subjects' dominant alpha frequency. The DFTs were calculated by using three-cycle-long data segments before stimulus onset (e.g., $10 \mathrm{~Hz}$ results in a $0.3 \mathrm{~s}$ window). Each data segment was Hanning tapered before the DFT was calculated. We estimated phase as close as possible to stimulus onset with a window of three cycles to give sensible frequency resolution under the assumption of phase continuity until frequency onset, since poststimulus-evoked activity would confound and contaminate the phase estimate. The trials in each subject were then binned according to where in the alpha cycle the stimulus onset occurred. These bins formed the basis for regressors used in the integrated EEG/fMRI analysis. Instantaneous amplitude was estimated based on the same prestimulus time windows used for the individual alpha frequency for both the real and the pseudo-trials.

Effects of phase on power. For the effect of phase on poststimulus power, we first computed the poststimulus power effects related to the stimulus presentation. Significant time-frequency clusters of increases or decreases were further tested for effects of phase using a one-way ANOVA. Time-frequency analysis was based on $0.5 \mathrm{~s}$ Hanning tapered windows in a $2-30 \mathrm{~Hz}$ frequency range in $0.05 \mathrm{~s}$ time steps up to $0.8 \mathrm{~s}$ poststimulus. Poststimulus power estimates were normalized by computing the logarithm of the ratio relative to a prestimulus baseline. The average power value in the period between 350 and $250 \mathrm{~ms}$ before stimulus onset served as a baseline.

\section{fMRI data acquisition}

MRI data were acquired using a 3.0-T whole-body MRI scanner (Magnetom TIM Trio; Siemens) and a custom-built eight-channel array coil 
(Stark Contrast) covering the occipital cortex for functional imaging. Twenty-one slices positioned parallel to the calcarine sulcus were recorded using a BOLD gradient echo EPI sequence [repetition time (TR), $1400 \mathrm{~ms}$; echo time (TE), $30 \mathrm{~ms} ; 60^{\circ}$ flip angle; $3.0 \mathrm{~mm}$ slice thickness; 0.5 $\mathrm{mm}$ gap; voxel size, $3.5 \times 3.5 \times 3.5 \mathrm{~mm}$; with bias field correction filter].

After the functional scans, two anatomical scans of the entire brain were acquired, one with the eight-channel occipital array coil (3D MPRAGE; TE, $3.5 \mathrm{~ms}$; TR, $2300 \mathrm{~ms} ; 10^{\circ}$ flip angle; 192 slices per slab; voxel size $0.8 \times 0.8 \times 0.8 \mathrm{~mm}$; with bias field correction filter) and one with an eight-channel phased array head coil (3D MPRAGE; TE, $3.0 \mathrm{~ms}$; TR, $2300 \mathrm{~ms} ; 8^{\circ}$ flip angle; 192 slices; voxel size $1.0 \times 1.0 \times 1.0 \mathrm{~mm}$; with bias field correction filter). The anatomical image from the occipital array coil was acquired because the bias field in sensitivity is similar to the functional images and as a result provides a better match for coregistration.

\section{Image preprocessing}

We preprocessed the (f)MRI data using statistical parametric mapping software (SPM8; http://www.fil.ion.ucl.ac.uk/spm/software); preprocessing involved realignment, correction for motion and differences in slice acquisition time, spatial normalization, and smoothing with an isotropic Gaussian kernel of $6 \mathrm{~mm}$ full-width at half-maximum. Anatomical normalization to MNI space was performed by coregistration of the functional images, first with the anatomical T1 scan acquired with the eight channels occipital array coil, which has the same bias field as the functional images. Second, this anatomical image was coregistered with the anatomical scan obtained with an eight-channel head coil, keeping the functional images in alignment. Parameters for the normalization to MNI space were estimated by normalizing this scan to the T1 MNI template provided by SPM8, and were subsequently applied to all anatomical and functional images.

\section{Stimulus-related BOLD response}

For the standard analysis, a general linear model was constructed. Stick functions convolved with the canonical hemodynamic response function from SPM8 were used to model the stimulus onset, onsets of reported color changes of the fixation stimulus, and fixation change misses. The contrast estimate images for the wedge-shaped visual stimulation onsets and the color change of the central fixation were subjected to a single sample $t$ test at group level.

\section{Integrated EEG-fMRI analysis}

Influence of alpha amplitude on the evoked BOLD response. The effect of prestimulus alpha amplitude on the evoked BOLD signal was assessed using a finite impulse response (FIR) model as implemented in SPM8. Activity levels were estimated at a resolution of $1.4 \mathrm{~s}$ (one value per TR) in a time range starting $2.8 \mathrm{~s}$ prestimulus and ending $16.8 \mathrm{~s}$ poststimulus $(14$ time points in total). Based on a median split, all visual stimulation trials were sorted into two conditions with either high or low prestimulus alpha amplitude. Since occipital alpha power has been reported to show a (negative) intrinsic correlation with BOLD signal in visual areas, we also modeled pseudo-trials without stimulation after applying the same median split. The FIR model estimates were extracted and averaged for right hemispheric regions of interest that we defined by the overlap of stimulus-related activity ( $p<0.05$, uncorrected at group level) with Brodmann areas 17, 18, and 19 from the WFU Pick Atlas (Maldjian et al., $2003,2004)$. We then tested for an interaction between the factors trial type (two levels: real and pseudo) and amplitude (two levels: high and low). Such an interaction would implicate a genuine effect of alpha amplitude on the evoked BOLD response. In practice, this interaction effect was tested by first subtracting the FIR time courses for high and low pseudo-trials from high and low real trials, respectively, followed by a cluster-based nonparametric statistical test based on a dependent samples $t$ test (see Statistical inference, below).

Influence of alpha phase on the evoked BOLD response. A similar FIR model as for amplitude was constructed for the phase effect. In this case, four phase bins were constructed for the estimated alpha phase at stimulus onset, as follows: $-\pi$ to $-0.5 \pi$ (trough fall, bin 1 ), $-0.5 \pi$ to 0 (trough rise, bin 2), 0 to $0.5 \pi$ (peak rise, bin 3 ), and $0.5 \pi$ to $\pi$ (peak fall, bin 4 ). This model did not contain pseudo-trials. Further model specifi- cations were the same as for the amplitude effect. Significance was assessed using a cluster-based nonparametric statistical test based on the $F$ statistic obtained by a one-way ANOVA (phase with four levels).

For comparison of the results with analysis on channel level, we constructed a model based on channel-level alpha phase estimates. For this analysis, phase was estimated for each subject at the right posterior channel with the largest absolute mixing weight for the selected right posterior alpha component. The other steps in the analysis were the same as for the analysis based on the right posterior alpha component.

\section{Statistical inference}

The effects of alpha phase and amplitude on fMRI and EEG responses were assessed using a nonparametric cluster-based randomization procedure that effectively controls the type 1 error rate in a situation involving multiple comparisons (Maris and Oostenveld, 2007). This randomization procedure allows for the construction of user-defined test statistics tailored to the effect of interest within the framework of a cluster-based randomization test. It works by calculating the sum of a statistic of choice over all data points within a cluster that exceeds an arbitrary predefined threshold. Each cluster sum is then compared with a distribution of maximum cluster sums obtained by randomly permuting the labels of the independent variable. The number of randomizations for computing the reference distribution was 1000 .

For the effect of alpha amplitude on the BOLD response, we used the $t$ value obtained by the dependent-samples $t$ test between the high and low alpha amplitude BOLD time courses (after subtraction of the pseudotrials). Clusters were defined as adjacent data points exceeding an uncorrected $p$ value of 0.1 for this $t$ test (e.g., data points that fall within the upper or lower $5 \%$ of the $t$ distribution).

For the phase effect on the BOLD response, we used the $F$ value obtained from a repeated-measures ANOVA with four levels as a test statistic. Clusters were defined as data points that exceeded an uncorrected threshold of 0.1 for the data points ranging between 0 and $10 \mathrm{~s}$ poststimulus. Post hoc differences between phase bins were assessed with $t$ tests on the average BOLD response within the significant clusters.

Clustering for the power analysis was performed over both time and frequency dimensions and for the event-related potential (ERP) only over the time dimension. For the time frequency analysis of power and the ERP, a two-tailed one-sample $t$ test against baseline served as the test statistic. Clusters were defined as adjacent data points passing a $p=0.1$ uncorrected threshold. Randomization was performed by randomly multiplying the single subject effects by 1 or -1 . For the ERP, separate one-tailed $t$ tests were performed for predefined intervals for the P1 ( $80-120 \mathrm{~ms}$ poststimulus) and $\mathrm{N} 1$ ( $150-200 \mathrm{~ms}$ poststimulus). The ERP was root-mean-square normalized from $200 \mathrm{~ms}$ before stimulus to 1000 $\mathrm{ms}$ poststimulus before statistical tests were performed.

\section{Results}

All subjects performed close to ceiling on the incidental behavioral task involving the central fixation point. They detected its color change in at least $97.9 \%$ of occurrences, which means they made maximally one mistake across the six task blocks. Average reaction time was $771 \mathrm{~ms}$ with a SD across subjects of $94 \mathrm{~ms}$. Neither alpha phase $\left(F_{(3,45)}=1.69, p=0.18\right)$ nor amplitude $\left(t_{(15)}=\right.$ $0.57, p=0.57)$ had any significant effect on reaction times to the fixation change.

For 16 of the 17 subjects, a right lateralized posterior alpha component could be identified from the recordings of ongoing EEG activity. The grand average power spectrum is depicted in Figure $2 A$ and contains a distinct peak in the alpha frequency range. The average scalp topography of the mixing weights (Fig. $2 B$ ) shows a clear right posterior lateralization of the modeled activity. Dipole fits for these 16 subjects (Fig. 2C) tentatively link this component to activity in right hemispheric early visual areas. Phase and amplitude of this component were analyzed trial by trial to probe their impact on evoked responses. 
A

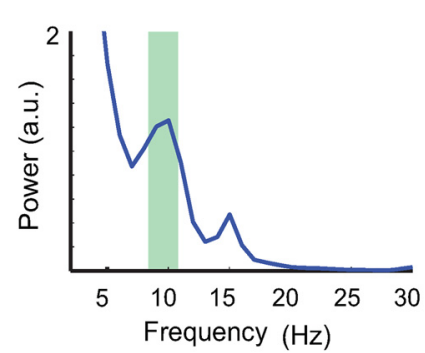

B

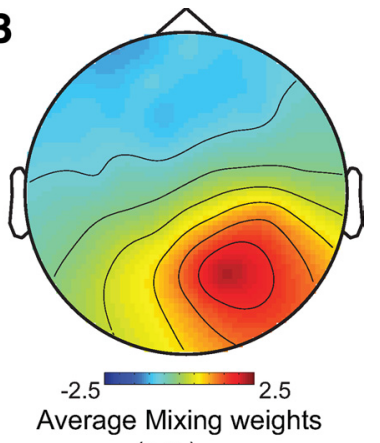

(a.u.)

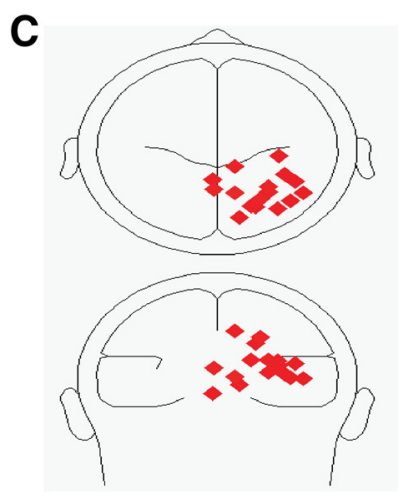

likov and Schiff, 2003; Mazaheri and Jensen, 2006; Sauseng et al., 2007; Ritter and Becker, 2009).

\section{Evoked fMRI responses}

The peripheral experimental $17 \mathrm{~ms}$ visual stimulus in the left lower quadrant of the visual field yielded a significant BOLD response in predominantly right hemisphere visual regions (Fig. $1 B$ ), whereas the central fixation change resulted in bilateral activation throughout a large part of the visual system (Fig. 1C). We extracted data from three regions of interest [Boadmann area (BA) 17-19] to test for effects of prestimulus alpha amplitude and phase on fMRI signal related to the peripheral stimulus.

For amplitude, clear differences between high and low amplitude were present in the estimated FIR time courses (Fig. 4A-C). Yet, these differences could be attributed to a negative intrinsic relation between alpha amplitude and the BOLD signal that is already present without external stimulation. After subtracting the FIR model estimates for the high and low alpha amplitude pseudo-trials from the high and low amplitude real trials, there was no significant effect of alpha amplitude on the evoked BOLD response (Fig. $4 D-F$ ).

Contrary to alpha amplitude, a significant effect of alpha phase on the evoked BOLD response was observed for Brodmann area 17 ( $p=0.013$, corrected for multiple comparisons) and a trend for Brodmann area 18 ( $p=0.063$, corrected) (Fig. 5A-C). Subsequent $t$ tests for differences between the different phase bins showed that visual stimuli presented at the trough of the alpha cycle produce a larger BOLD response than stimuli presented at its peak (Fig. $5 D, E$ ). This demonstrates that the strength of the neuronal response, as expressed in the amplitude of the BOLD response, is dependent on the phase of the ongoing alpha rhythm at the onset of stimulation.

To illustrate the effect of selecting the right posterior alpha independent component, we performed the same analysis for phase estimated at the channel level with the maximal absolute mixing weight. For this channel, however, no significant modulation of the BOLD signal by alpha phase could be observed (Fig. $5 F-H)$. This suggests the ICA decomposition greatly improves estimation of the right posterior alpha signal.

\section{Discussion}

The objective of our study was to test whether ongoing alpha oscillations generate cyclic gain changes of evoked neural responses. We used the technique of simultaneously recording EEG and fMRI (Laufs et al., 2008) to address this question, because the BOLD signal permits a well localized assessment of the amount of synaptic activity induced by a stimulus (Logothetis, 2008). We analyzed signal from stimulus-sensitive regions of interest in the right hemisphere and observed a relatively weaker evoked BA17 (and perhaps BA18) BOLD response when the ultra-short stimulus in the peripheral left hemifield was presented at the peak of the alpha wave compared with its trough. We conclude from these findings that the position in the phase cycle of the posterior alpha oscillation impacts on the overall strength of the neural evoked response, as indexed by the BOLD signal.

In addition to investigating the effect of alpha phase on the evoked BOLD response, we also interrogated our data for potential effects related to alpha amplitude. Although trials preceded by higher alpha amplitude were indeed, as one might predict, associated with lower evoked BOLD responses, we believe this effect to be accounted for by an intrinsic negative correlation between posterior alpha power and occipital BOLD signal (Goldman et al., 2002; Gonçalves et al., 2006; Laufs et al., 2006; Ritter et al., 
A

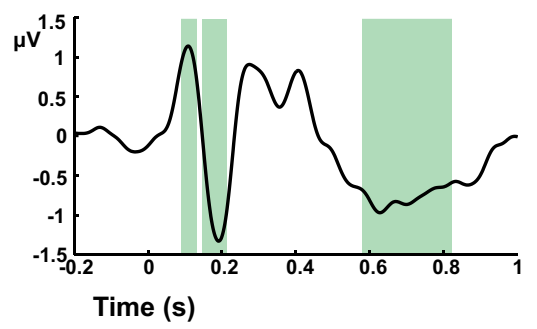

C

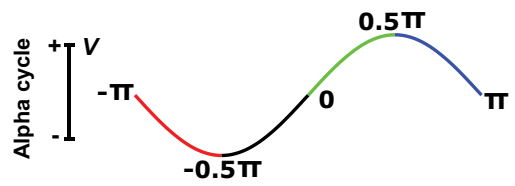

B

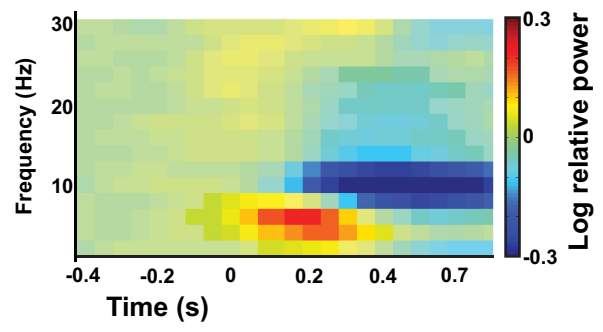

D

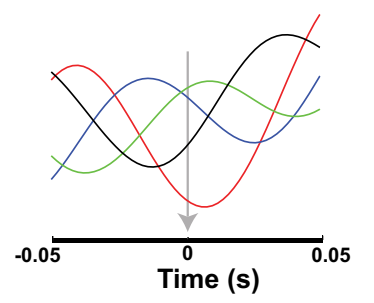

Figure 3. Stimulus-induced EEG responses. $\boldsymbol{A}$, Grand average potential in response to the wedge stimulus for the selected component back-projected to scalp level evoked. The shaded area depicts significant deviations from zero. $\boldsymbol{B}$, Time-frequency representation of power for the selected component relative to a prestimulus baseline. Highlighted time-frequency clusters are significant after correction for multiple comparisons. $\boldsymbol{C}$, The color coding of the phase bins used for sorting of the EEG trials. D, The phase-sorted evoked potentials of a representative subject, with the arrow indicating stimulus onset.

\section{A FIR time-courses}

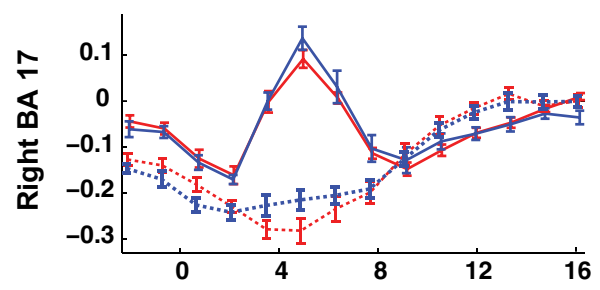

B

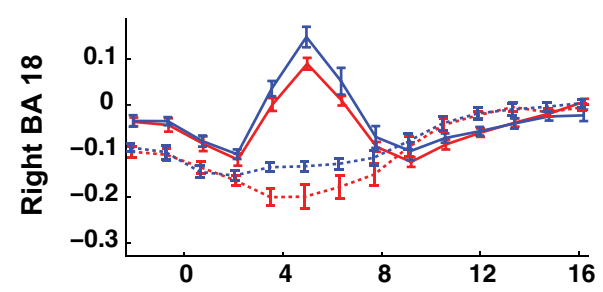

C

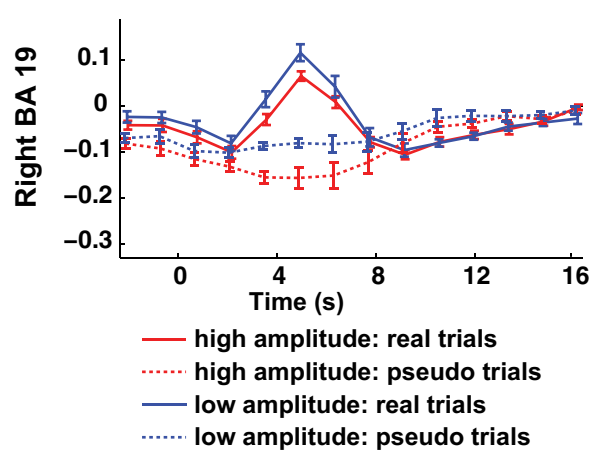

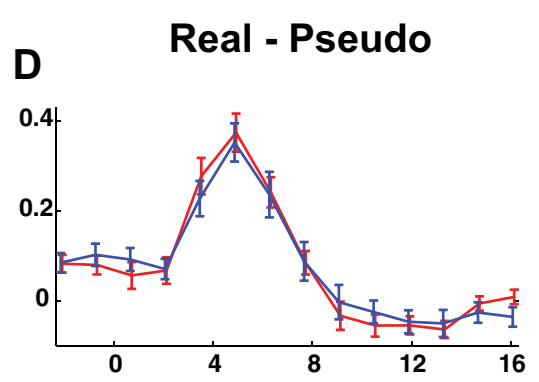

E

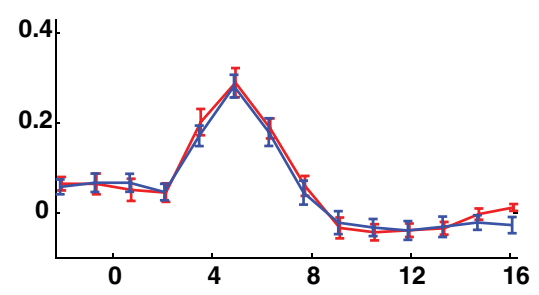

$\mathbf{F}$

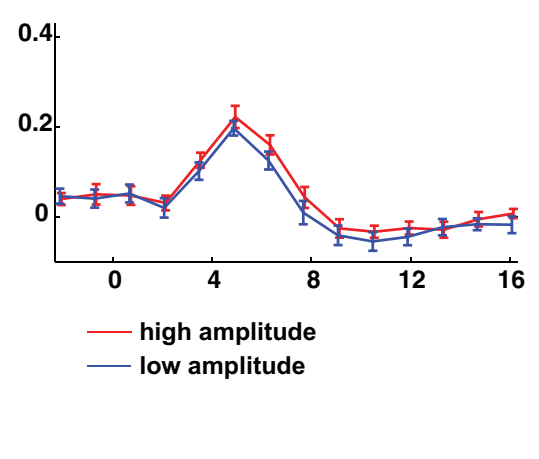

Figure 4. Evoked BOLD responses for high and low prestimulus alpha amplitude. $\boldsymbol{A}-\boldsymbol{C}$, FIR time courses for both pseudo and real trials for both high and low prestimulus alpha amplitude for right hemispheric regions of interest in $B A 17,18$, and 19. $\boldsymbol{D}-\boldsymbol{F}$, FIR signal time courses after pseudo-trial time courses have been subtracted from high and low amplitude trials. Error bars indicate SEM.

2009; Scheeringa et al., 2009; Yuan et al., 2010). When we removed effects from this intrinsic correlation, we retained no evidence for an effect of alpha amplitude on the strength of the evoked response.

At first glance, it might seem somewhat surprising that we observed an effect of phase but not of amplitude. One possible explanation is that alpha amplitude is more variable across the cortex than alpha phase, especially in the presence of a central attention-demanding behavioral task. If posterior alpha oscillations are coordinated by synchronous thalamic drive or thalamocortical feedback, then alpha phase could be expected to be largely homogenous across the visual cortex. Conversely, the actual strength of the alpha oscillations at different places across the visual cortex might be more variable because of local intervening processes, which may be poorly reflected in the component signal we used to determine amplitude. An alternate possible explanation is a (nonlinear) threshold effect of alpha amplitude on evoked responses. This possibility could be tested in futures studies by using a range of stimuli with varying strengths and it might also be relevant for the relative size of the effect that phase has on the evoked response.

Interestingly, this effect of alpha phase on the evoked BOLD response was not reflected in a modulation of EEG power changes in the lower frequencies. This could suggest that the BOLD response covers synaptic activity changes that are not captured in the surface EEG signal. Residual gradient artifacts present in simultaneous EEG/fMRI measurements prevented us from testing for potential effects in frequencies $>30 \mathrm{~Hz}$ that might carry such information (Logothetis, 2008). The effects of alpha phase on the conventional ERP response are hard to assess because the sorting and averaging based on phase introduces phase effects in the ERP even in absence of a stimulus (Klimesch et al., 2009; Risner et al., 2009; Ritter and Becker, 2009). We can therefore only speculate that no such relationship was present in our data given that there was no effect of prestimulus alpha phase on the power of the poststimulus frequencies (i.e., the spectral representation of the ERPs).

Our observation that alpha oscillations reflect periodic variations in cortical excitability and hence neural response strength is compatible with existing views that alpha oscillations serve the cyclic inhibition of task-irrelevant regions (Klimesch et al., 2007; Mazaheri and Jensen, 
ICA component

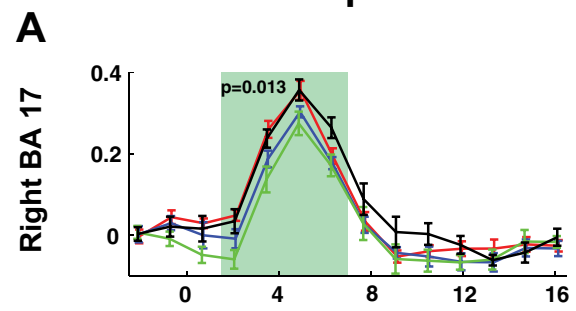

B

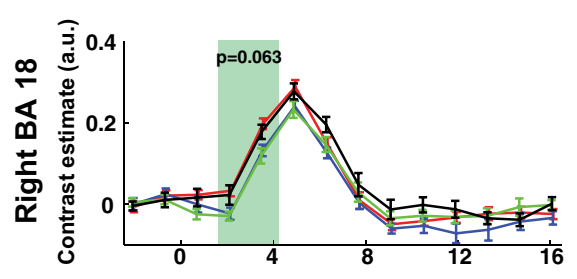

C

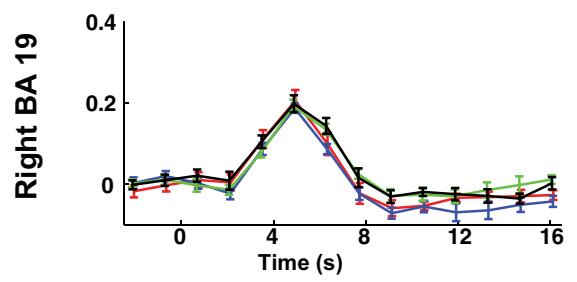

ICA component

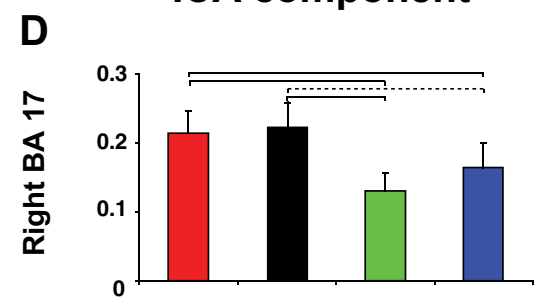

E
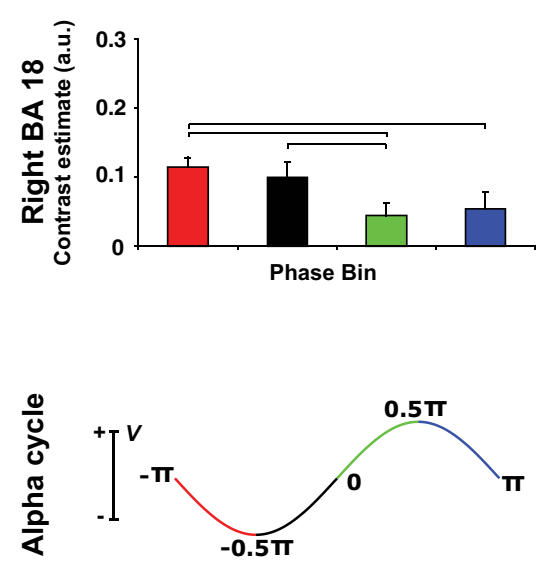

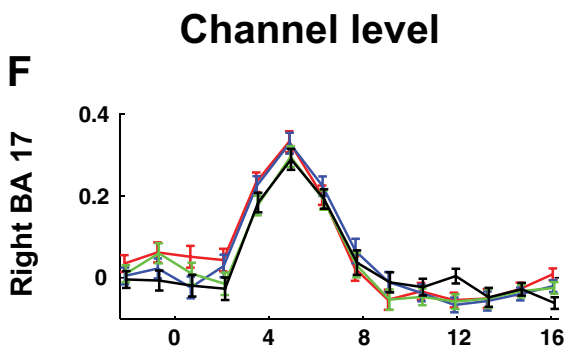

G

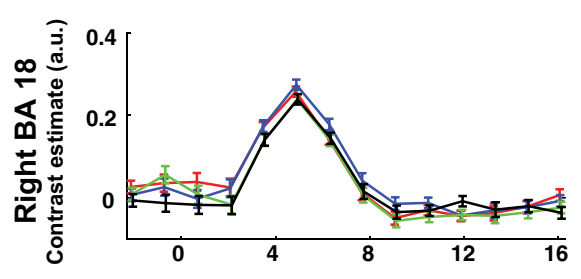

H

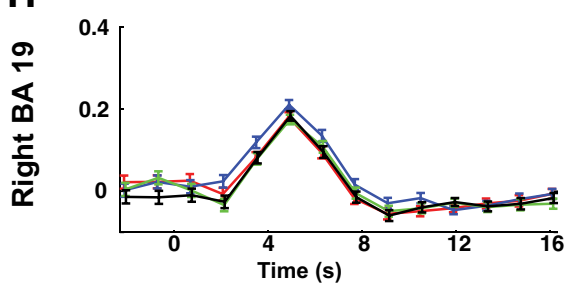

Figure 5. Effect of alpha phase on the visual evoked BOLD response in right hemispheric regions of interest. $\boldsymbol{A}-\boldsymbol{C}$, BOLD responses for the four phase bins in regions BA 17,18 , and 19 for the selected right posterior alpha component. The shaded area indicates when there is a significant effect of prestimulus alpha phase on the BOLD response after correction for multiple comparisons. $D$, $\boldsymbol{E}$, Differences between the four phase bins for the BOLD responses in BA 17 and 18 for the analysis based on the right posterior alpha component. The bars indicate the average contrast estimates over the significant time points. $\boldsymbol{F}-\boldsymbol{H}$ correspond to $\boldsymbol{A}-\boldsymbol{C}$, but are based on data with phase binning from signal at the channel with the absolute mixing weight for the individual right posterior alpha component. Significance was assessed using two-sided dependent sample $t$ tests. Solid horizontal lines indicate effects at $p<0.05$, dashed lines indicate $0.05<p<0.1$. Error bars are SEM. Color coding of phase position is according to the bottom of the middle column.

2010). In line with this, recent studies have reported that both perceptual performance on periliminal stimuli (Busch et al., 2009; Mathewson et al., 2009) and perception of phosphenes evoked by transcranial magnetic stimulation depend on alpha phase (Romei et al., 2008b). The modulation of the BOLD response by alpha phase was strongest in putative BA17, and was present to a lesser extent in BA18, which suggests that alpha phase modulates the neural response in early sensory cortices, providing a neural substrate for the perceptual effects described above. A potential caveat is that source location for alpha oscillations is not sufficiently precise to assign the component we analyzed to specific visual areas. Furthermore, the BOLD response is not fast enough to distinguish the initial stimulus-driven neural response from feedback-related activity (e.g., whether the alpha component reflected activity in areas providing such feedback instead of in V1/V2). Our results show that stimuli presented at the trough of the alpha cycle produce a larger BOLD response compared with those presented at the peak. If alpha oscillations yield cyclic inhibition, then this inhibition appears to occur at the peak for the posterior EEG alpha rhythm. Such a "neuronic shutter" (Nunn and Osselton, 1974) or "rhythmic pulsing" (Mazaheri and Jensen, 2010) could provide a functionally appealing mechanism for clearing and updating of incoming information. In a recent study, we have argued that sustained alertness could rely on such a windshield-wiper effect of large-scale, synchronized alpha activity, which in turn might be orchestrated by central cognitive control regions (Sadaghiani et al., 2010).
The question of the physiological mechanism behind an alpha cycle-related modulation of the BOLD response arises. We are not aware of any evidence suggesting that such an effect could be considered an artifact related to a cyclic modulation of the neurovascular coupling function. In other words, we consider our findings, which are grounded in the BOLD signal, to indicate that neural responses to stimulation are modulated by the alpha cycle. GABAergic feedback from interneurons has been strongly implicated in the physiological mechanism generating the alpha rhythm (Lopes da Silva et al., 1976; Crunelli and Leresche, 1991; Jones et al., 2000; Liley et al., 2002). Thus, it is possible that rhythmic neuronal activity generating the alpha oscillations is a consequence of a GABAergic inhibitory feedback paced by neocortical or thalamic rhythm generators (Hughes and Crunelli, 2005; Lörincz et al., 2008, 2009; Liley et al., 2010). We suggest that this GABAergic feedback could function to directly dampen processing by providing shunting inhibition to pyramidal neurons or reduce the efficacy of incoming excitatory input. The phase of the alpha oscillations then reflects the cyclic state of this inhibition.

More generally, our findings demonstrate that the phase of oscillations in postsynaptic potentials can potentially serve as a mechanism of gain control for incoming neural activity, as put forward by theories on neural communication (Fries, 2005). Our study illustrates how such phase-related gain effects on neural processing can be studied noninvasively in human subjects by means of simultaneously recording EEG and fMRI. Methodolog- 
ically, this study provides a proof of principle, since this technique can be adapted easily to investigate other combinations of frequency, scalp site, and brain regions of interest across a range of different cognitive states.

\section{References}

Allen PJ, Polizzi G, Krakow K, Fish DR, Lemieux L (1998) Identification of EEG events in the MR scanner: the problem of pulse artifact and a method for its subtraction. Neuroimage 8:229-239.

Allen PJ, Josephs O, Turner R (2000) A method for removing imaging artifact from continuous EEG recorded during functional MRI. Neuroimage 12:230-239.

Barry RJ, de Pascalis V, Hodder D, Clarke AR, Johnstone SJ (2003) Preferred EEG brain states at stimulus onset in a fixed interstimulus interval auditory oddball task, and their effects on ERP components. Int J Psychophysiol 47:187-198.

Bechtereva NP, Zontov VV (1962) The relationship between certain forms of potentials and the variations in brain excitability (based on EEG, recorded during photic stimuli triggered by rhythmic brain potentials). Electroencephalogr Clin Neurophysiol 14:320-330.

Bell AJ, Sejnowski TJ (1995) An information-maximization approach to blind separation and blind deconvolution. Neural Comput 7:1129-1159.

Berg P, Scherg M (1994) A fast method for forward computation of multiple-shell spherical head models. Electroencephalogr Clin Neurophysiol 90:58-64.

Berger H (1929) Über das Elektrenkephalogramm des Menschen. Arch f Psychiatr 87:527-570.

Bishop GH (1932) Cyclic change in the excitability of the optic pathway of the rabbit. Am J Physiol 130:213-224.

Busch NA, Dubois J, VanRullen R (2009) The phase of ongoing EEG oscillations predicts visual perception. J Neurosci 29:7869-7876.

Callaway E 3rd, Yeager CL (1960) Relationship between reaction time and electroencephalographic alpha phase. Science 132:1765-1766.

Crunelli V, Leresche N (1991) A role for GABAB receptors in excitation and inhibition of thalamocortical cells. Trends Neurosci 14:16-21.

Debener S, Ullsperger M, Siegel M, Engel AK (2006) Single-trial EEG-fMRI reveals the dynamics of cognitive function. Trends Cogn Sci 10:558-563.

Delorme A, Makeig S (2004) EEGLAB: an open source toolbox for analysis of single-trial EEG dynamics including independent component analysis. J Neurosci Methods 134:9-21.

Dustman RE, Beck EC (1965) Phase of alpha brain waves, reaction time and visually evoked potentials. Electroencephalogr Clin Neurophysiol 18:433-440

Fries P (2005) A mechanism for cognitive dynamics: neuronal communication through neuronal coherence. Trends Cogn Sci 9:474-480.

Goldman RI, Stern JM, Engel J Jr, Cohen MS (2002) Simultaneous EEG and fMRI of the alpha rhythm. Neuroreport 13:2487-2492.

Gonçalves SI, de Munck JC, Pouwels PJ, Schoonhoven R, Kuijer JP, Maurits NM, Hoogduin JM, Van Someren EJ, Heethaar RM, Lopes da Silva FH (2006) Correlating the alpha rhythm to BOLD using simultaneous EEG/ fMRI: inter-subject variability. Neuroimage 30:203-213.

Hughes SW, Crunelli V (2005) Thalamic mechanisms of EEG alpha rhythms and their pathological implications. Neuroscientist 11:357-372.

Jokisch D, Jensen O (2007) Modulation of gamma and alpha activity during a working memory task engaging the dorsal or ventral stream. J Neurosci 27:3244-3251.

Jones SR, Pinto DJ, Kaper TJ, Kopell N (2000) Alpha-frequency rhythms desynchronize over long cortical distances: a modeling study. J Comput Neurosci 9:271-291.

Klimesch W, Sauseng P, Hanslmayr S (2007) EEG alpha oscillations: the inhibition-timing hypothesis. Brain Res Rev 53:63-88.

Klimesch W, Sauseng P, Gruber W (2009) The functional relevance of phase reset: a comment to Risner et al (2009): The visual evoked potential of surface alpha rhythm phase. Neuroimage 47:5-7.

Kruglikov SY, Schiff SJ (2003) Interplay of electroencephalogram phase and auditory-evoked neural activity. J Neurosci 23:10122-10127.

Laufs H, Holt JL, Elfont R, Krams M, Paul JS, Krakow K, Kleinschmidt A (2006) Where the BOLD signal goes when alpha EEG leaves. Neuroimage 31:1408-1418.

Laufs H, Daunizeau J, Carmichael DW, Kleinschmidt A (2008) Recent advances in recording electrophysiological data simultaneously with magnetic resonance imaging. Neuroimage 40:515-528.
Liley DT, Cadusch PJ, Dafilis MP (2002) A spatially continuous mean field theory of electrocortical activity. Network 13:67-113.

Liley DT, Bojak I, Dafilis MP, van Veen L, Frascoli F, Foster BL (2010) Bifurcations and state changes in the human alpha rhythm: theory and experiment. In: Modeling phase transitions in the brain (Steyn-Ross DA, Steyn-Ross M, eds). New York: Springer.

Logothetis NK (2008) What we can do and what we cannot do with fMRI. Nature 453:869-878.

Lopes da Silva FH, van Rotterdam A, Barts P, van Heusden E, Burr W (1976) Models of neuronal populations: the basic mechanisms of rhythmicity. Prog Brain Res 45:281-308.

Lörincz ML, Crunelli V, Hughes SW (2008) Cellular dynamics of cholinergically induced alpha $(8-13 \mathrm{~Hz})$ rhythms in sensory thalamic nuclei in vitro. J Neurosci 28:660-671.

Lörincz ML, Kékesi KA, Juhász G, Crunelli V, Hughes SW (2009) Temporal framing of thalamic relay-mode firing by phasic inhibition during the alpha rhythm. Neuron 63:683-696.

Makeig S, Westerfield M, Jung TP, Enghoff S, Townsend J, Courchesne E, Sejnowski TJ (2002) Dynamic brain sources of visual evoked responses. Science 295:690-694.

Maldjian JA, Laurienti PJ, Kraft RA, Burdette JH (2003) An automated method for neuroanatomic and cytoarchitectonic atlas-based interrogation of fMRI data sets. Neuroimage 19:1233-1239.

Maldjian JA, Laurienti PJ, Burdette JH (2004) Precentral gyrus discrepancy in electronic versions of the Talairach atlas. Neuroimage $21: 450-455$.

Maris E, Oostenveld R (2007) Nonparametric statistical testing of EEG- and MEG-data. J Neurosci Methods 164:177-190.

Mathewson KE, Gratton G, Fabiani M, Beck DM, Ro T (2009) To see or not to see: prestimulus alpha phase predicts visual awareness. J Neurosci 29:2725-2732.

Mazaheri A, Jensen O (2006) Posterior alpha activity is not phase-reset by visual stimuli. Proc Natl Acad Sci U S A 103:2948-2952.

Mazaheri A, Jensen O (2008) Asymmetric amplitude modulations of brain oscillations generate slow evoked responses. J Neurosci 28:7781-7787.

Mazaheri A, Jensen O (2010) Rhythmic pulsing: linking ongoing brain activity with evoked responses. Front Hum Neurosci 4:177.

Medendorp WP, Kramer GF, Jensen O, Oostenveld R, Schoffelen JM, Fries P (2007) Oscillatory activity in human parietal and occipital cortex shows hemispheric lateralization and memory effects in a delayed double-step saccade task. Cereb Cortex 17:2364-2374.

Nunn CM, Osselton JW (1974) The influence of the EEG alpha rhythm on the perception of visual stimuli. Psychophysiology 11:294-303.

Rihs TA, Michel CM, Thut G (2007) Mechanisms of selective inhibition in visual spatial attention are indexed by alpha-band EEG synchronization. Eur J Neurosci 25:603-610.

Risner ML, Aura CJ, Black JE, Gawne TJ (2009) The visual evoked potential is independent of surface alpha rhythm phase. Neuroimage 45:463-469.

Ritter P, Becker R (2009) Detecting alpha rhythm phase reset by phase sorting: caveats to consider. Neuroimage 47:1-4.

Ritter P, Moosmann M, Villringer A (2009) Rolandic alpha and beta EEG rhythms' strengths are inversely related to fMRI-BOLD signal in primary somatosensory and motor cortex. Hum Brain Mapp 30:1168-1187.

Romei V, Rihs T, Brodbeck V, Thut G (2008a) Resting electroencephalogram alpha-power over posterior sites indexes baseline visual cortex excitability. Neuroreport 19:203-208.

Romei V, Brodbeck V, Michel C, Amedi A, Pascual-Leone A, Thut G (2008b) Spontaneous fluctuations in posterior alpha-band EEG activity reflect variability in excitability of human visual areas. Cereb Cortex 18:2010-2018.

Sadaghiani S, Scheeringa R, Lehongre K, Morillon B, Giraud AL, Kleinschmidt A (2010) Intrinsic connectivity networks, alpha oscillations, and tonic alertness: a simultaneous electroencephalography/functional magnetic resonance imaging study. J Neurosci 30:10243-10250.

Sauseng P, Klimesch W, Gruber WR, Hanslmayr S, Freunberger R, Doppelmayr M (2007) Are event-related potential components generated by phase resetting of brain oscillations? A critical discussion. Neuroscience 146:1435-1444.

Scheeringa R, Petersson KM, Oostenveld R, Norris DG, Hagoort P, Bastiaansen MC (2009) Trial-by-trial coupling between EEG and BOLD identifies networks related to alpha and theta EEG power in- 
creases during working memory maintenance. Neuroimage 44: $1224-1238$.

Shah AS, Bressler SL, Knuth KH, Ding M, Mehta AD, Ulbert I, Schroeder CE (2004) Neural dynamics and the fundamental mechanisms of eventrelated brain potentials. Cereb Cortex 14:476-483.

Thut G, Théoret H, Pfennig A, Ives J, Kampmann F, Northoff G, PascualLeone A (2003) Differential effects of low-frequency rTMS at the occipital pole on visual-induced alpha desynchronization and visual-evoked potentials. Neuroimage 18:334-347.

Thut G, Nietzel A, Brandt SA, Pascual-Leone A (2006) Alpha-band electroencephalographic activity over occipital cortex indexes visuospatial attention bias and predicts visual target detection. J Neurosci 26:9494-9502.

van Dijk H, Schoffelen JM, Oostenveld R, Jensen O (2008) Prestimulus oscillatory activity in the alpha band predicts visual discrimination ability. J Neurosci 28:1816-1823.

van Dijk H, van der Werf J, Mazaheri A, Medendorp WP, Jensen O (2010) Modulations in oscillatory activity with amplitude asymmetry can pro- duce cognitively relevant event-related responses. Proc Natl Acad Sci U S A 107:900-905.

Varela FJ, Toro A, John ER, Schwartz EL (1981) Perceptual framing and cortical alpha rhythm. Neuropsychologia 19:675-686.

Wyart V, Sergent C (2009) The phase of ongoing EEG oscillations uncovers the fine temporal structure of conscious perception. J Neurosci 29:12839-12841.

Yeung N, Bogacz R, Holroyd CB, Cohen JD (2004) Detection of synchronized oscillations in the electroencephalogram: an evaluation of methods. Psychophysiology 41:822-832.

Yuan H, Liu T, Szarkowski R, Rios C, Ashe J, He B (2010) Negative covariation between task-related responses in alpha/beta-band activity and BOLD in human sensorimotor cortex: an EEG and fMRI study of motor imagery and movements. Neuroimage 49:2596-2606.

Zhang Y, Wang X, Bressler SL, Chen Y, Ding M (2008) Prestimulus cortical activity is correlated with speed of visuomotor processing. J Cogn Neurosci 20:1915-1925. 